May 2004

\title{
Effects of Fermion Masses and Twisting on Non-Integrable Phases on Compact Extra Dimensions
}

\author{
Müge Boz $^{a}$ and Namık K. Pak ${ }^{b}$ \\ ${ }^{a}$ Department of Physics, Hacettepe University, Turkey, TR06800 \\ ${ }^{b}$ Department of Physics, Middle East Technical University, Turkey, TR06531
}

\begin{abstract}
The effective potential for the Wilson loop in the $\mathrm{SU}(2)$ gauge theory with $N_{f}$ massive fundamental and $N_{a}$ massive adjoint fermions on $S^{1} \times M^{4}$ is computed at the one-loop level, assuming periodic boundary conditions for the gauge field and general boundary conditions for fermions. It is shown that there are critical values for the bare mass, and the boundary condition parameter for the adjoint fermions, beyond which the symmetry pattern changes. However, neither bare mass, nor the boundary condition parameter for the fundamental fermion play any role on the vacuum structure, thus the symmetry breaking pattern. When the two different types of fermions with equal masses exist together the pattern of the fundamental fermion dominate, and $\mathrm{SU}(2)$ gauge symmetry remains intact independent of the fermion masses.
\end{abstract}




\section{Introduction}

It has long been known that gauge theories on multiply-connected spaces exhibit anomalous behavior in that the gauge connection is promoted to a physical observable. The simplest example is provided by Aharonov-Bohm effect [1] according to which the interference of matter waves in the presence of an impenetrable domain of magnetic field is modulated by the magnetic flux. This observation has later been furthered [2] to prove the dynamical nature of the connection and the irrelevance of single-valuedness of the matter and gauge fields. The analysis of [2], which was focused on massless fermions in the fundamental representation of the gauge group, has subsequently been generalized to adjoint fermions $[3,4,5]$. Recently, effects of adjoint fermion masses have been incorporated into the previous works [6], and it was pointed out that there exist certain critical values of the fermion masses accross which the symmetries of the system change.

For appreciating the importance of the Wilson loop dynamics, consider for definiteness a gauge theory in a 5-dimensional factorizable geometry $M^{4} \times S^{1}$ where $S^{1}$ is a circle with radius $R$. The gauge field $A_{B}(B=(\mu, y), \mu=0,1,2,3)$ has five independent components, and it is forbidden to have any local potential due to higher dimensional gauge invariance. However, the non-integrable phase factor

$$
\theta(x)=-i \ln \left[P e^{i \int_{y=0}^{y=2 \pi R} d y A_{y}\left(x_{\mu}, y\right)}\right]
$$

being inherently non-local in the direction of extra dimension, develops a non-local potential in the presence of charged bulk fields. In case $\theta(x)$ develops a nonvanishing vacuum expectation value (VEV) the gauge symmetry can be broken dynamically depending on the model parameters [5]. This has been particularly useful in string compactifications [7]. Furthermore, recently it has been pointed out that radiatively-lifted vanishing potential for the non-integrable phase factor $\theta(x)$ makes it a perfect candidate for inflaton $[8,9,10]$ which has to acquire an extremely flat potential to comply with the requirements of successful inflation.

In this work, we consider a non-supersymmetric $\mathrm{SU}(2)$ gauge model with $N_{f}$ massive adjoint fermions, and $N_{a}$ massive fundamental fermions, with the most general boundary condition parameters for the fermions and the gauge fields on $S^{1} \times M^{4}$. Here is a brief summary of the present work in relation with the previous works:

Hosotani has previously considered a SU(2) gauge theory defined both on $S^{1} \times R^{1}$ and $S^{1} \times M^{3}$ with massless fermions, but with arbitrary boundary condition (bc) parameters for the gauge fields, and fermions [5]. He has shown that the $\mathrm{SU}(2)$ gauge symmetry is not broken, when the fermions are in the fundamental representation (FR), irrespective of the values of the bc parameters ${ }^{1}$. But the SU(2) symmetry breaks down to U(1) for certain values of the bc parameter below a certain critical value, for the adjoint representation (AR). Takenaga more recently considered an $\mathrm{SU}(2)$ gauge theory $S^{1} \times M^{4}$ with massive adjoint fermions, with periodic boundary conditions for fermions [6]. He has shown that below a certain critical value of the bare mass the symmetry again breaks down to $\mathrm{U}(1)$.

In Section 3, we considered a $\mathrm{SU}(2)$ gauge theory with massive adjoint fermions and with arbitrary bc parameters. We have shown that below certain critical values of the bare mass and bc parameter the symmetry breaks down to $\mathrm{U}(1)$, and agrees with the results of Hosotani and Takenaga, respectively, in the corresponding limits.

\footnotetext{
${ }^{1}$ In more detail, for $\delta_{f}<\pi / 2$, absolute minimum is located at $\theta_{m}=\pi$, corresponding to $U^{s y m}=-I$, and for $\pi / 2<\delta_{f}<\pi$, the absolute minimum is located at $\theta_{m}=0$, corresponding to $U^{s y m}=I$. Both of these $U^{s y m}$ are elements of the center of $\mathrm{SU}(2)$, thus the $\mathrm{SU}(2)$ symmetry is unbroken.
} 
In Section 4, we extended this discussion in [5] for massless fundamental fermions, by including bare masses for fermions. We have shown that neither bc parameter $\delta_{f}$, nor the bare mass for the fundamental fermion play any role on the vacuum structure/symmetry breaking pattern.

Finally, in Section 5, we considered the general case with $N_{f}$ fundamental and $N_{a}$ adjoint fermions with equal masses. We have observed that the fundamental fermions play a more dominant role than the adjoint ones, on the gauge symmetry pattern, as the result turns out to be very similar to the pure fundamental fermions case.

\section{The Effective Potential}

Consider an $\mathrm{SU}(2)$ gauge theory on $M^{4} \times S^{1}$ with $N_{a}$ adjoint and $N_{f}$ fundamental fermions. The action is completely fixed by gauge invariance

$$
S=\int d^{4} x d y\left[-\frac{1}{2 g_{5}^{2}} \operatorname{tr}\left\{F_{A B} F^{A B}\right\}+\bar{\psi}\left(\gamma^{A} D_{A}-m_{f}\right) \psi+\bar{\lambda}\left(\gamma^{A} D_{A}-m_{a}\right) \lambda\right],
$$

where $\psi$ and $\lambda$ stand, respectively, for fundamental and adjoint fermion fields with masses $m_{f}$ and $m_{a}$, and $g_{5}$, with dimension of (mass $)^{-1 / 2}$, is the higher dimensional gauge coupling. The potential for $A_{5}$ is perfectly flat since gauge invariance forbids the induction of any local operator which can lift the flatness. However, the phase of the Wilson loop $\theta(x)$ is inherently non-local in the extra dimension and thus it can acquire a non-trivial non-local potential. Indeed, the gauge field kinetic term in (2), after dimensional reduction, generates the kinetic term

$$
\mathcal{L}_{K K}^{(4)}=\frac{1}{2 L^{2} g_{4}^{2}} \sum_{a}\left(\partial_{\mu} \theta^{a}\right)^{2}
$$

where $g_{4}=\frac{g_{5}}{\sqrt{2 \pi R}}$ is the four dimensional gauge coupling constant, and we defined a new field such that

$$
\theta^{a}(x)=g_{5} \int_{0}^{2 \pi R} d y A^{5, a}(x, y)=2 \pi R g_{5} A^{5, a}(x)=L g_{5} A^{5, a}(x)
$$

using the compactness of $S^{1}$ which guarantees the $y$-independence of the zero mode $A^{5, a}(x, y)$. However, the exactly flat potential of (3) is lifted by the gauge boson and fermion loops. This radiative contribution, denoted by $V_{a f}$, is given by

$$
\begin{aligned}
V_{a f}\left(\theta, N_{a}, N_{f}, z_{a}, z_{f}, \delta_{a}, \delta_{f}\right) & =\frac{1}{c_{1}}\left\{-3 \sum_{n=1} \frac{1}{n^{5}}[1+\cos 2 n \theta]\right. \\
& +2 N_{a} \sum_{n=1} \frac{F\left(z_{a} n\right)}{n^{5}}\left[2 \cos n \delta_{a}+\cos n\left(2 \theta+\delta_{a}\right)+\cos n\left(2 \theta-\delta_{a}\right)\right] \\
& \left.+2 N_{f} \sum_{n=1} \frac{F\left(z_{f} n\right)}{n^{5}}\left[\cos n\left(\theta+\delta_{f}\right)+\cos n\left(\theta-\delta_{f}\right)\right]\right\}
\end{aligned}
$$

where

$$
F(z n)=e^{-z n}\left[1+z n+\frac{1}{3} z^{2} n^{2}\right] \quad \text { with } \quad z=m L
$$

and

$$
\frac{1}{c_{1}}=\frac{3}{2 \pi^{2} L^{5}}
$$


Here, the $\theta$ is related to $\theta^{a}$ in Eq. (4), through the relationship:

$$
L g_{5} A_{5}^{a} \tau^{a}=\theta^{a} \tau^{a}=C\left(\begin{array}{cc}
\theta & 0 \\
0 & -\theta
\end{array}\right) C^{+},
$$

with the constant $2 \times 2$ matrix satisfying $C^{+} C=I$, and $\theta=\sqrt{\theta_{1}^{2}+\theta_{2}^{2}+\theta_{3}^{2}}$. We would like to point out, following Hosotani [5], that because of the invariance of the boundary conditions under global gauge transformations the effective potential does not depend on $C$, and for the $S U(2)$ case depends only on the single $\theta$ and the phases of the fermions.

In (5), the first line follows from the gauge and the ghost fields, and the second and the third lines are the contributions of $N_{a}$ massive adjoint and $N_{f}$ massive fundamental fermions, respectively. The expression (5) reduces correctly to various special cases already discussed in the literature $[2,3,4,5,6]$. One notes that the phases $\delta_{a}$, and $\delta_{f}$ are defined through the boundary conditions. As $S^{1}$ is not simply connected, boundary conditions must be specified for the single valuedness of the observables. For the gauge field we adopt periodic boundary conditions; for the fermion fields we impose the following general boundary conditions:

$$
\begin{aligned}
& \psi_{f}(x, y+L)=e^{i \delta_{f}} \psi_{f}(x, y) \\
& \psi_{a}(x, y+L)=e^{i \delta_{a}} \psi_{a}(x, y) .
\end{aligned}
$$

In what follows we discuss the three specific cases of adjoint, fundamental and adjoint plus fundamental fermions separately. In each case we analyze the potential landscape both analytically and numerically with the aim of determining if the original gauge symmetry is respected by the effective potential (5).

\section{The case with Adjoint Fermions only}

We first consider the case which there are $N_{a}$ massive adjoint fermions with the phases $\delta_{a}$. The effective potential takes the form

$$
V_{a}\left(\theta, N_{a}, z_{a}, \delta_{a}\right)=\frac{1}{c_{1}} \sum_{n=1} \frac{1}{n^{5}}\left[-3+4 N_{a} F\left(z_{a} n\right) \cos n \delta_{a}\right][1+\cos 2 n \theta],
$$

Note that the effective potential reduces to that of $N_{a}$ massless adjoint fermions with phases $\delta_{a}$ when $z \rightarrow 0$ [5], and differs from the model considered by Takenaga [6] by the $\cos n \delta_{a}$ term multiplying $F_{a}$.

To identify the role played by the massive fermions with phase $\delta_{a}$ on the vacuum structure, we have to look at the two special limits, namely $z_{a} \rightarrow \infty$, and $z_{a} \rightarrow 0$. The behaviour in the first case is identical to that of Takenaga [6], as the fermion is decoupled in this case. The dominant contribution comes from the gauge sector, and the vacuum configuration is given by $\theta=0[\bmod \pi]$ independent of $\delta_{a}$. Clearly, the $\mathrm{SU}(2)$ gauge symmetry is not broken in this case. In the massless limit, $z_{a} \rightarrow 0$, let us note that when $\delta_{a}=0$, the vacuum configuration is given by $\theta=\pi / 2[\bmod \pi]$ [4]. However when $\delta_{a} \neq 0$, we will see that there exists a critical value $\delta_{a}^{c}$, above which $\theta=0[\bmod \pi]$ is an absolute minimum. To find the critical value $\delta_{c}^{(a)}$, we define:

$$
c_{2} V_{a}^{\prime \prime}\left(\theta=0[\bmod \pi], N_{a}, z_{a}=0, \delta_{a}\right)=\sum_{n=1} \frac{1}{n^{3}}\left[3-4 N_{a} \cos n \delta_{a}\right]
$$


where

$$
c_{2}=\frac{c_{1}}{4}
$$

and we have used the standard definitions:

$$
\begin{aligned}
\sum \frac{\cos n \pi}{n^{D}} & =-\left[1-2^{(-D)}\right] \xi_{D}, \text { and } \\
\xi_{D} & =\sum \frac{1}{n^{D}},
\end{aligned}
$$

We plot $c_{2} V_{a}^{\prime \prime}\left(\theta=0[\bmod \pi], N_{a}=1, z_{a}=0, \delta_{a}\right)$ with respect to $\delta_{a}$ in Figure 1 . As can be seen from Figure 1 that there is a critical value at $\delta_{a}^{c_{1}}=0.53$.

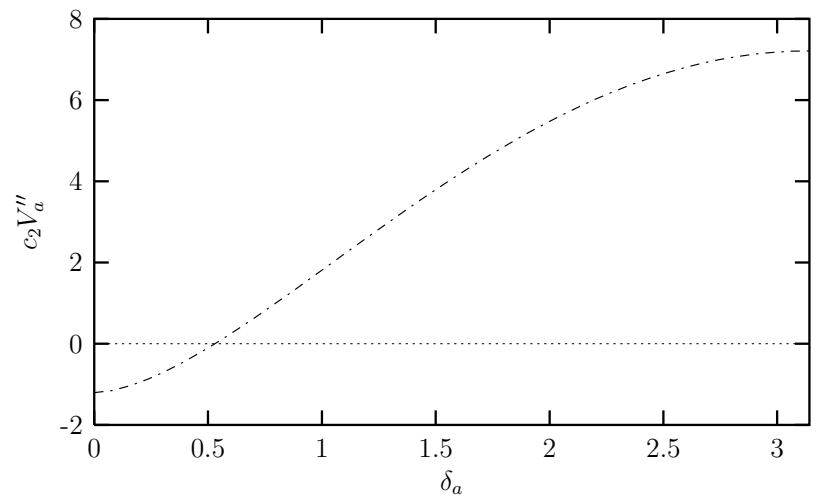

Figure 1: The $\delta_{a}$ dependence of $c_{2} V_{a}^{\prime \prime}\left(\theta=0[\bmod \pi], N_{a}=1, z_{a}=0, \delta_{a}\right)$.

Consistency with the results of Davies and McLachan [4] requires that there must be a critical value (same or different than $\delta_{a}^{c_{1}}$ ) below which $\theta=\pi / 2[\bmod \pi]$ is an absolute minimum. To find this critical value, we again define

$$
c_{2} V_{a}^{\prime \prime}\left(\theta=\pi / 2[\bmod \pi], N_{a}, z_{a}=0, \delta_{a}\right)=\sum_{n=1} \frac{(-1)^{n}}{n^{3}}\left[3-4 N_{a} \cos n \delta_{a}\right],
$$

and in Figure 2, we investigate the dependence of $c_{2} V_{a}^{\prime \prime}\left(\theta=\pi / 2[\bmod \pi], N_{a}=1, z_{a}=0, \delta_{a}\right)$ on $\delta_{a}$.

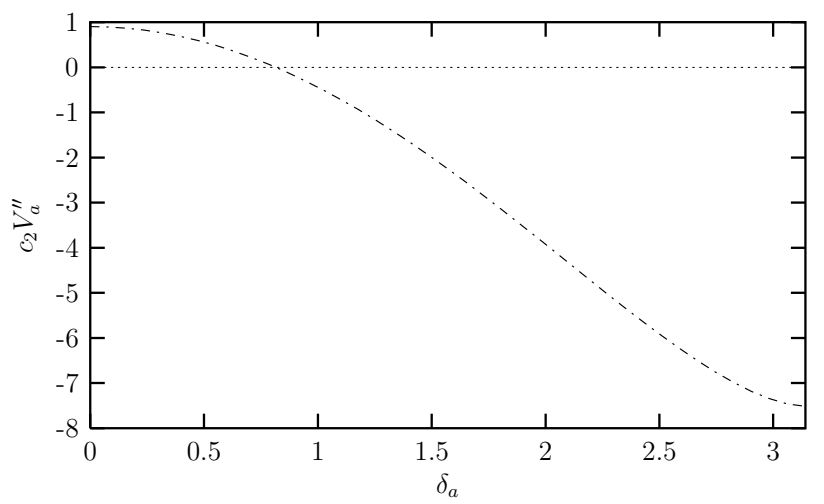

Figure 2: The $\delta_{a}$ dependence of $c_{2} V_{a}^{\prime \prime}\left(\theta=\pi / 2[\bmod \pi], N_{a}=1, z_{a}=0, \delta_{a}\right)$.

As can be seen from Figure 2 that there is a critical point at $\delta_{a}^{c_{2}}=0.81$, which is different from the previous case. 
If we summarize,

(i) For $\delta_{a}^{c_{2}}<\delta<\pi, \theta=0[\bmod \pi]$ is an absolute minimum, and the $\mathrm{SU}(2)$ gauge symmetry is not broken.

(ii) For $0<\delta_{a}<\delta_{a}^{c_{1}}, \theta=\pi / 2[\bmod \pi]$ is an absolute minimum, and the gauge symmetry is dynamically broken down to $\mathrm{U}(1)$.

This observation suggests that there must exist certain critical values of $z_{a}$ at which gauge symmetry breaking patterns change when $0<\delta_{a}<\delta_{a}^{c_{1}}$ (note that this does not happen when $\delta_{a}^{c_{2}}<\delta_{a}<\pi$, as there is no difference in the symmetry breaking structure from $z_{a} \rightarrow \infty$ to $\left.z_{a} \rightarrow 0\right)$.

Before adressing the stability question of the vacuum configurations identified above, we would like to study the interval $\delta_{a}^{c_{1}}<\delta_{a}<\delta_{a}^{c_{2}}$ in detail. Figure 1 and Figure 2 suggest that in this interval of $\delta_{a}$ all the three vacuum configurations, namely $\theta=0, \pi / 2$ exist simultaneously. The behaviour of $c_{1} V_{a}\left(\theta, N_{a}=1, z_{a}=0, \delta_{a}\right)$ for different values of $\delta_{a}$ in this interval is plotted in Figure 3.

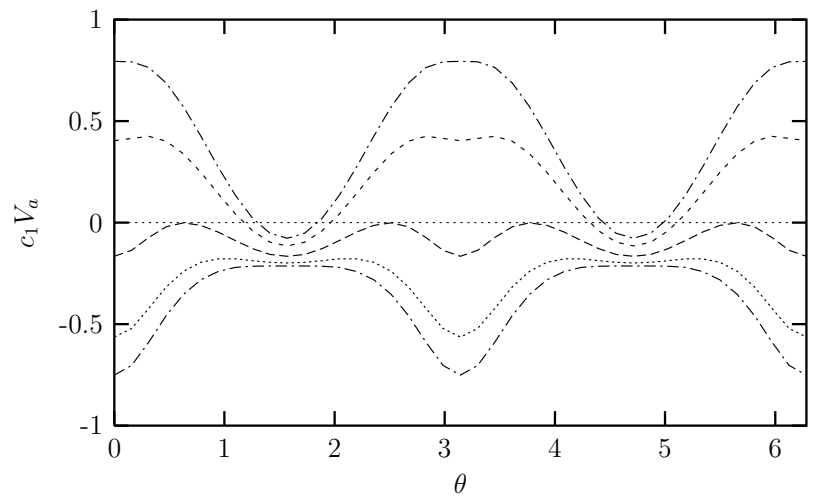

Figure 3: The dependence on $\theta$ of $c_{1} V_{a}\left(\theta, N_{a}=1, z_{a}=0, \delta_{a}\right)$. Here, the top and bottom dotdashed curves represent $\delta_{a}^{c_{1}}=0.53$, and $\delta_{a}^{c_{2}}=0.81$ values of $\delta_{a}$ respectively, whereas the three different curves in the $\delta_{a}^{c_{1}}<\delta_{a}<\delta_{a}^{c_{2}}$ interval correspond to $\delta_{a}=0.61,0.71,0.78$, from top to bottom, respectively.

We see from Figure 3 that for $\delta_{a}$ close to $\delta_{a}^{c_{2}}, \theta=0[\bmod \pi]$ and $\delta_{a}$ close to $\delta_{a}^{c_{1}}, \quad \theta=\pi / 2[\bmod \pi]$ are the absolute minima, respectively. Somewhere in between, namely at $\delta_{a}=0.71$, the two are degenerate. That is in the massless case, for the values of $\delta_{a}$ within the interval $\left(\delta_{a}^{c_{1}}, \delta_{a}^{c_{2}}\right)$, there exists a mixed phase, namely unbroken $\mathrm{SU}(2)$ phase together with the broken phase $\mathrm{U}(1)$. This interesting phenomena clearly deserves further study, which we postpone to a future work.

To confirm the existence of the critical values of $z_{a}$ we have to study the stability of the configurations $\theta=0[\bmod \pi]$, and and $\theta=\pi / 2[\bmod \pi]$ with respect to $z_{a}$, corresponding to vacuum configurations, in the limits $z_{a} \rightarrow \infty$ as well as $z_{a} \rightarrow 0$ (when $\delta_{a}^{c_{2}}<\delta_{a}<\pi$ ), and $z_{a} \rightarrow 0$ (when $0<\delta_{a}<\delta_{a}^{c_{1}}$ ), respectively.

The second derivative of the effective potential is plotted with respect to $z_{a}$ for $\theta=0[\bmod \pi]$ in Figure 4, and for $\theta=\pi / 2[\bmod \pi]$ in Figure 5 , for $N_{a}=1$, with their explicit expressions given as :

$$
\begin{aligned}
c_{2} V_{a}^{\prime \prime}\left(\theta=0[\bmod \pi], N_{a}, z_{a}, \delta_{a}^{c_{2}}<\delta_{a}<\pi\right) & =3 \xi_{3}-4 N_{a} \sum_{n=1} \frac{F\left(z_{a} n\right) \cos n \delta_{a}}{n^{3}} \\
c_{2} V_{a}^{\prime \prime}\left(\theta=\pi / 2[\bmod \pi], N_{a}, z_{a}, 0<\delta_{a}<\delta_{a}^{c_{1}}\right) & =-\frac{9}{4} \xi_{3}-4 N_{a} \sum_{n=1} \frac{(-1)^{n} F\left(z_{a} n\right) \cos n \delta_{a}}{n^{3}},
\end{aligned}
$$




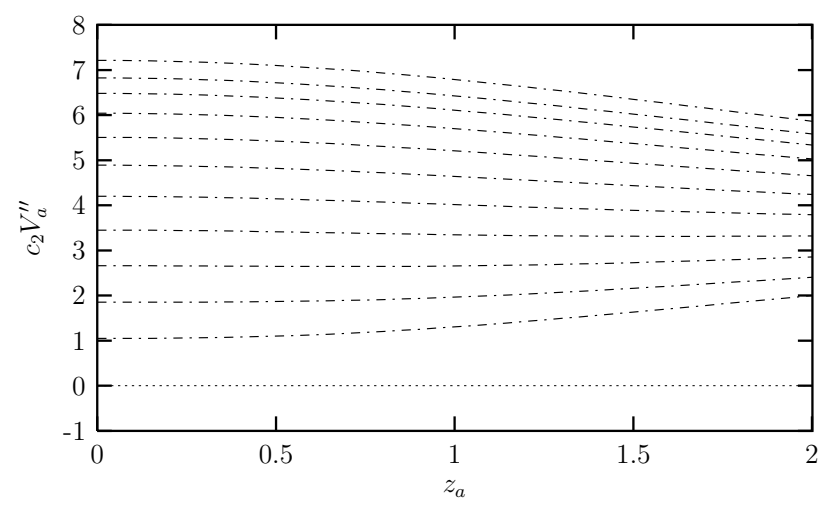

Figure 4: The $z_{a}$ dependence of $c_{2} V_{a}^{\prime \prime}\left(\theta=0[\bmod \pi], N_{a}=1, z_{a}, \delta_{a}^{c_{1}}<\delta_{a}<\pi\right)$. Here, $\delta_{a}^{c_{2}}=0.81$ for the bottom curve, whereas $\delta_{a}=\pi$ for the top curve.

We see from Figure 4 that $c_{2} V_{a}^{\prime \prime}\left(\theta=0, N_{a}=1, z_{a}, \delta_{a}^{c_{2}}<\delta<\pi\right)$ is always positive, and there is no critical value $z_{a}^{c}$ where $V_{a}^{\prime \prime}$ changes sign, and $\theta=0[\bmod \pi]$ is stable independent of $z_{a}$. This is consistent with the previous observation that as long as $\delta_{a}^{c_{2}}<\delta_{a}<\pi, \theta=0[\bmod \pi]$ is an absolute minimum both in $z_{a} \rightarrow \infty$ and $z_{a} \rightarrow 0$ limits.

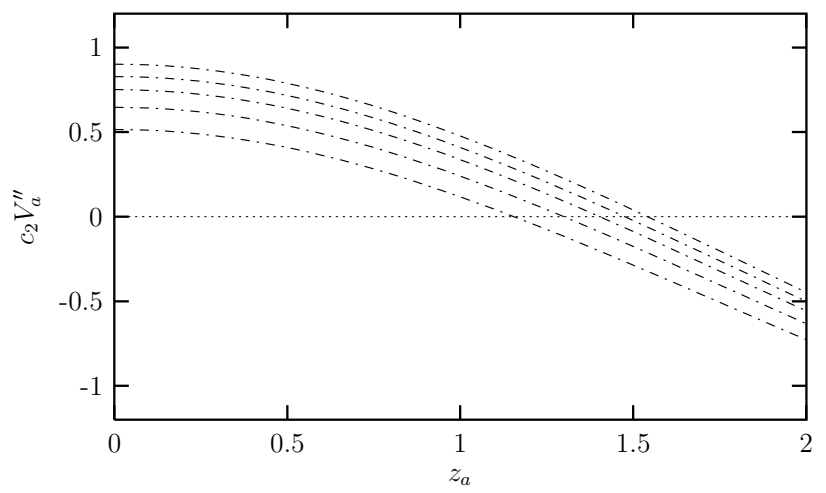

Figure 5: The $z_{a}$ dependence of $c_{2} V_{a}^{\prime \prime}\left(\theta=\pi / 2[\bmod \pi], N_{a}=1, z_{a}, 0<\delta<\delta_{a}^{c_{1}}\right)$. Here, $\delta_{a}=0$ for the top curve, whereas $\delta_{a}^{c_{1}}=0.53$ for the bottom curve.

We see from Figure 5 that there are critical values for $z_{a}$, depending on the values of $\delta_{a}$, below which $V_{a}^{\prime \prime}\left(\theta=\pi / 2, N_{a}=1, z_{a}, 0<\delta<\delta_{a}^{c_{1}}\right)$ is positive. The largest of these $z_{a}^{c}$ corresponding to $\delta_{a}=0$ is $z_{a}^{c}=1.5$, which is identical to the result of Takenaga [6]. The larger $\delta_{a}$ is, within the allowed range $\left(0, \delta_{a}^{c_{1}}\right)$, the smaller $z_{a}$ gets. That is, the symmetry breaking pattern is more sensitive to adjoint mass for larger values of the phase $\delta_{a}$, in the allowed range $\left(0, \delta_{a}^{c_{1}}\right)$.

If we summarize

(i) when $0<\delta_{a}<\delta_{a}^{c_{1}}, V_{a}^{\prime \prime}\left(\theta=\pi / 2, N_{a}=1, z_{a}, \delta_{a}\right)>0$ for a set of values for $z_{a}<z_{a}^{c}=1.5$, the gauge symmetry is broken to $\mathrm{U}(1)$,

(ii) when $\delta_{a}^{c_{2}}<\delta_{a}<\pi, V_{a}^{\prime \prime}\left(\theta=0[\bmod \pi], N_{a}=1, z_{a}, \delta_{a}\right)>0$, independent of the values of $z_{a}$, and the gauge symmetry $\mathrm{SU}(2)$ is intact.

(iii) when $\delta_{a}^{c_{2}}<\delta_{a}<\delta_{a}^{c_{1}}$, there exists a mixed phase, namely the unbroken $\mathrm{SU}(2)$ phase together with the broken phase $\mathrm{U}(1)$.

For instance, in Figure 6, we show the $z_{a}$ dependence of $c_{2} V_{a}^{\prime \prime}\left(\theta=\pi / 2[\bmod \pi], N_{a}=1, z_{a}, \delta_{a}\right)$ when $\delta_{a}=0.71$, for which case the two minima are degenerate (see Figure 3), with the critical 


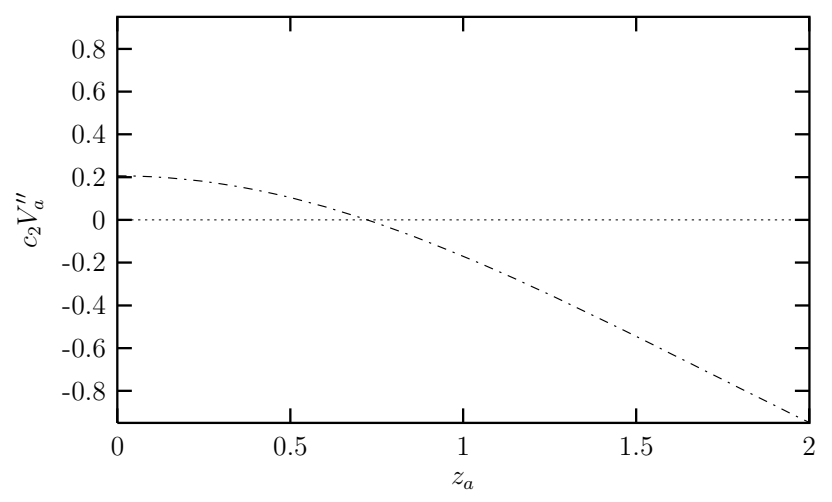

Figure 6: The $z_{a}$ dependence of $c_{2} V_{a}^{\prime \prime}\left(\theta=\pi / 2[\bmod \pi], N_{a}=1, z_{a}, \delta_{a}=0.71\right)$.

value $z_{c}=0.72$. We would have obtained identical information if we have plotted $c_{2} V_{a}^{\prime \prime}(\theta=$ $\left.0[\bmod \pi], N_{a}=1, z_{a}, \delta_{a}=0.71\right)$.

\section{The Case with Fundamental fermions only}

When there are $N_{f}$ massive fundamental fermions only, with the bc phases $\delta_{f}$, the potential takes the form:

$$
V_{f}\left(\theta, N_{f}, z_{f}, \delta_{f}\right)=\frac{1}{c_{1}} \sum_{n=1} \frac{1}{n^{5}}\left[-3(1+\cos 2 n \theta)+4 N_{f} F\left(z_{f} n\right) \cos n \delta_{f} \cos n \theta\right] .
$$

The effective potential reduces to that of $N_{f}$ massless fundamental fermions with phases $\delta_{f}[2,5]$ in the $z_{f} \rightarrow 0$ limit. Again, to identify the role played by the masses and the $\delta_{f}$-phases of the fermion on the vacuum structure, we have to look at the $z_{f} \rightarrow \infty$, and $z_{f} \rightarrow 0$ limits. As the fermions decouple in the former case, this case is identical to that of adjoint fermions, and the vacuum structure is given by $\theta=0[\bmod \pi]$ independently of $\delta_{f}$. The $\mathrm{SU}(2)$ gauge symmetry is intact in this regime.

Next, we look at the $z_{f} \rightarrow 0$ limit, in detail. First recall that this limit with $\delta_{f} \neq 0$ was considered by Hosotani [2], for $M^{3} \times S^{1}$. He has shown that the absolute minimum is $\theta=0$, for $\pi / 2<\delta_{f}<\pi$, and $\theta=\pi$ for $0<\delta_{f}<\pi / 2$ independent of the number of fermions $N_{f}$; furthermore these two absolute minima are degenerate for $\delta_{f}=\pi / 2$. He has further shown, as mentioned before in the footnote, that in both cases the gauge symmetry is unbroken. We have $M^{4} \times S^{1}$; therefore the critical value of $\delta_{f}\left(\delta_{f}^{c}\right)$, if there is any, could be different than that of Hosotani [2]. We first plot the expressions for $V_{f}^{\prime \prime}\left(\theta=0, N_{f}, z_{f}=0, \delta_{f}\right)$, and $V_{f}^{\prime \prime}\left(\theta=\pi, N_{f}, z_{f}=0, \delta_{f}\right)$, to identify the $\delta_{f}$-regions where $\theta_{m}=0, \pi$ are the minima, respectively.

Using (16), we get:

$$
\begin{aligned}
c_{2} V_{f}^{\prime \prime}\left(\theta=0, N_{f}, z_{f}=0, \delta_{f}\right) & =\sum_{n=1} \frac{1}{n^{3}}\left[3-N_{f} \cos n \delta_{f}\right], \\
c_{2} V_{f}^{\prime \prime}\left(\theta=\pi, N_{f}, z_{f}=0, \delta_{f}\right) & =\sum_{n=1} \frac{1}{n^{3}}\left[3-N_{f}(-1)^{n} \cos n \delta_{f}\right],
\end{aligned}
$$

and analyze the dependence on $\delta_{f}$ of $c_{2} V_{f}^{\prime \prime}\left(\theta=0, N_{f}, z_{f}=0, \delta_{f}\right)$ and $c_{2} V_{f}^{\prime \prime}\left(\theta=\pi, N_{f}, z_{f}=0, \delta_{f}\right)$ in Figure 7 and Figure 8, respectively when $N_{f}=1,2,3,4$. 


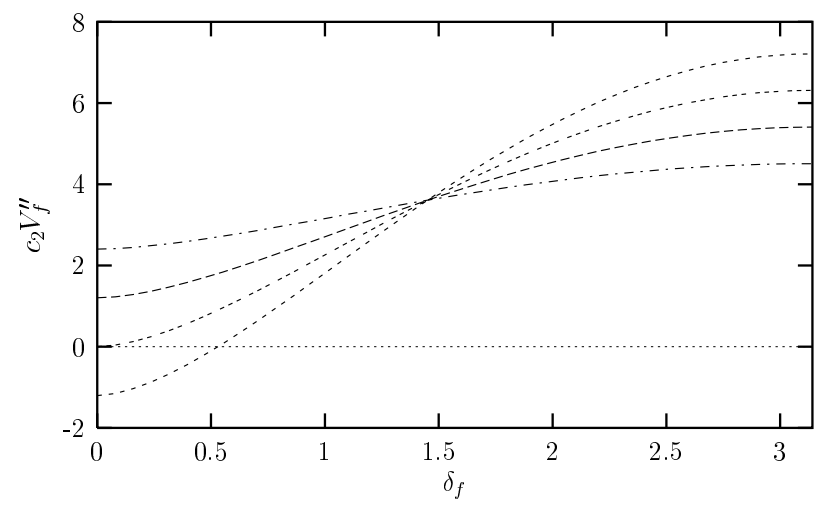

Figure 7: The $\delta_{f}$ dependence of $c_{2} V_{f}^{\prime \prime}\left(\theta=0, N_{f}, z_{f}=0, \delta_{f}\right)$, when $N_{f}=1$ (top curve), and $N_{f}=4$ (bottom curve). The curves in between are for $N_{f}=2, N_{f}=3$, from top to bottom.

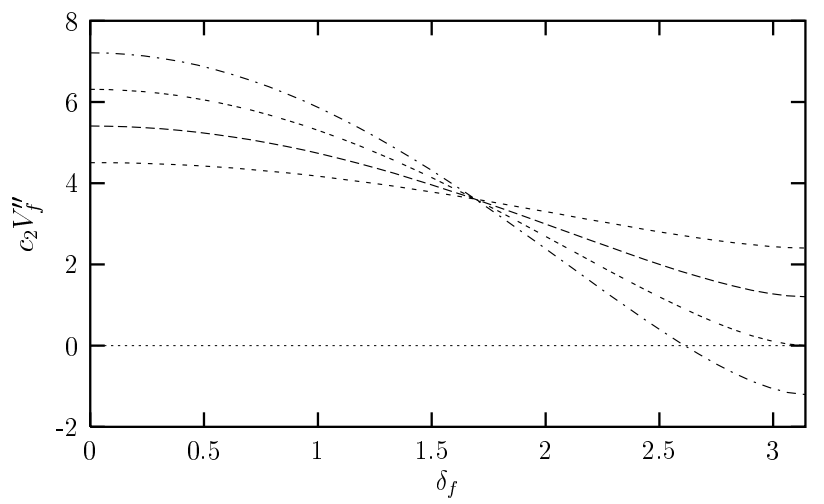

Figure 8: The $\delta_{f}$ dependence of $c_{2} V_{f}^{\prime \prime}\left(\theta=\pi, N_{f}, z_{f}=0, \delta_{f}\right)$, when $N_{f}=1$ (bottom curve) and $N_{f}=4$ (top curve). The curves in between are for $N_{f}=2, N_{f}=3$, from bottom to top.

A comparative look at Figure 7 and Figure 8 suggests that $c_{2} V_{f}^{\prime \prime}\left(\theta=0, N_{f}, z_{f}=0, \delta_{f}\right)$ and $c_{2} V_{f}^{\prime \prime}\left(\theta=\pi, N_{f}, z_{f}=0, \delta_{f}\right)$ do not change sign with the variation of $\delta_{f}$ for $N_{f}=1$, 2, whereas they change sign for $N_{f}=4$ (the bottom curve in Figure 7, and the top curve in Figure 8).

To verify that $N_{f}$ indeed plays a role on the structure of the minima, we study the sign of $V_{f}^{\prime \prime}\left(\theta, N_{f}, z_{f}=0, \delta_{f}\right)$ at the limits of $\delta_{f}$ for each $\theta=0$, and $\theta=\pi$ as a function of $N_{f}(\theta=0, \pi$ corresponding to the limits of periodic, and antiperiodic boundary conditions):

$$
\begin{aligned}
& c_{2} V_{f}^{\prime \prime}\left(\theta=0, N_{f}, z_{f}=0, \delta_{f}=0\right)=\left\{\left(3-N_{f}\right) \xi_{3}\right\}>0 \text { for } N_{f}=1,2, \\
& <0 \text { for } N_{f} \geq 4, \\
& c_{2} V_{f}^{\prime \prime}\left(\theta=\pi, N_{f}, z_{f}=0, \delta_{f}=\pi\right)=\left\{\left(3-N_{f}\right) \xi_{3}\right\}>0 \text { for } N_{f}=1,2, \\
& <0 \text { for } N_{f} \geq 4 \text {, }
\end{aligned}
$$

and

$$
\begin{aligned}
& c_{2} V_{f}^{\prime \prime}\left(\theta=0, N_{f}, z_{f}=0, \delta_{f}=\pi\right)=\left\{\frac{3}{4} \xi_{3}\left(N_{f}+4\right)\right\}>0 \text { for all } N_{f}, \\
& c_{2} V_{f}^{\prime \prime}\left(\theta=\pi, N_{f}, z_{f}=0, \delta_{f}=0\right)=\left\{\frac{3}{4} \xi_{3}\left(N_{f}+4\right)\right\} \gtrsim 0 \text { for all } N_{f} .
\end{aligned}
$$


One notices that for $N_{f} \geq 4$ case there are some subtleties, and thus we pay special attention to $N_{f}=4$ :

We see from Figure 7 that that there is a critical value $\delta_{a}^{c_{1}}=0.53$, above which $c_{2} V^{\prime \prime}(\theta=$ $\left.0, N_{f}, z_{f}=0, \delta_{f}=0\right)>0$. Moreover as Figure 8 suggests there is another critical value $\delta_{a}^{c_{2}}=2.61$ which is different from the former case, below which $c_{2} V_{f}^{\prime \prime}\left(\theta=\pi, N_{f}, z_{f}=0, \delta_{f}=0\right)>0$. Thus, for $N_{f}=4$, the absolute minima are:

$$
\begin{aligned}
& 0<\delta_{f}<\delta_{f}^{c_{1}}, \theta_{m}=\pi \\
& \delta_{f}^{c_{2}}<\delta_{f}<\pi, \theta_{m}=0 .
\end{aligned}
$$

However, as mentioned above for $\theta_{m}=0, \pi, U^{s y m}=(I,-I)$ and those lie in the center of $\mathrm{SU}(2)$, thus the symmetry is not broken.

Next, we would like to determine the regions of $\delta_{f}$, in which $\theta=0, \pi$ are the absolute minima, respectively. For this purpose, in Figure 10, and Figure 11, we have plotted $c_{1} V_{f}\left(\theta, N_{f}=1, z_{f}=\right.$ $\left.0, \delta_{f}\right)$, with respect to $\theta$, for selected values of $\delta_{f}$, in the $0<\delta_{f}<\pi / 2$, and $\pi / 2<\delta_{f}<\pi$ intervals, respectively.

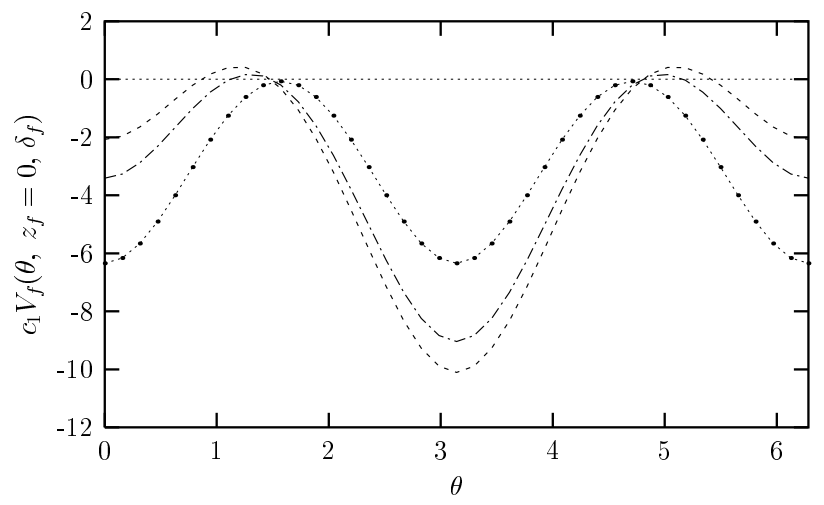

Figure 9: The dependence on $\theta$ of $c_{1} V_{f}\left(\theta, N_{f}=1, z_{f}=0,0<\delta_{f}<\pi / 2\right)$. Here, $\delta_{f}=0$, and $\delta_{f}=\pi / 4$ for the bottom and the middle curves, respectively, whereas $\delta_{f}=\pi / 2$ for the top curve.

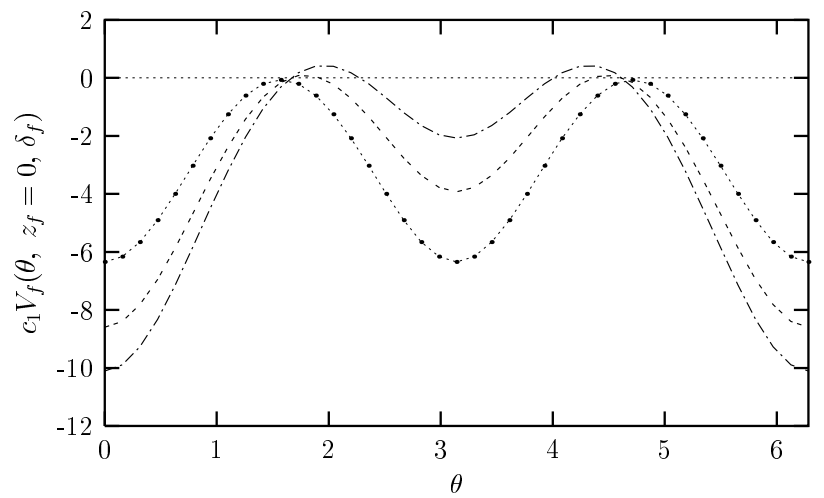

Figure 10: The dependence on $\theta$ of $c_{1} V_{f}\left(\theta, N_{f}=1, z_{f}=0, \pi / 2<\delta_{f}<\pi\right)$. Here, $\delta_{f}=7 \pi / 10$ and $\delta_{f}=\pi$ for the middle and top curves, respectively, whereas $\delta_{f}=\pi / 2$ for the bottom curve.

We see from Figure 9, and 10 that $\theta=0, \pi$ are the absolute minima for $\pi / 2<\delta_{f}$, and $\pi / 2<\delta_{f}<\pi$ respectively, as in the case discussed by Hosotani [2], and they are degenerate at 
$\delta_{f}=\pi / 2$. However, for $\theta_{m}=0, \pi, U^{\text {sym }}=(I,-I)$ and these lie in the center of $\mathrm{SU}(2)$, thus the symmetry is not broken.

Similar pattern can be obtained for the behaviour of $V_{f}\left(\theta, N_{f}=4, z_{f}=0, \delta_{f}\right)$ case. We have observed that the secondary local minima become shallower (that is smoothed out), however, with increasing $N_{f}$.

Next, we have to check the stability properties of the absolute minima under the variation of $V_{f}^{\prime \prime}\left(\theta, N_{f}, z_{f}, \delta_{f}\right)$ with respect to $z_{f}$. Using (16), we get:

$$
\begin{aligned}
& c_{2} V_{f}^{\prime \prime}\left(\theta=0, N_{f}, z_{f}, \pi / 2<\delta_{f}<\pi\right)=\sum_{n=1} \frac{1}{n^{3}}\left[3-N_{f} F\left(z_{f} n\right) \cos n \delta_{f}\right], \\
& c_{2} V_{f}^{\prime \prime}\left(\theta=\pi, N_{f}, z_{f}, 0<\delta_{f}<\pi / 2\right)=\sum_{n=1} \frac{1}{n^{3}}\left[3-(-1)^{n} N_{f} F\left(z_{f} n\right) \cos n \delta_{f}\right],
\end{aligned}
$$

and look for the critical values of $z_{f}$, where $c_{2} V_{f}^{\prime \prime}\left(\theta=0, N_{f}, z_{f}, \pi / 2<\delta_{f}<\pi\right)$, and $c_{2} V_{f}^{\prime \prime}(\theta=$ $\pi, N_{f}, z_{f}, 0<\delta_{f}<\pi / 2$ ) change sign, in Figure 11 and Figure 12, respectively, when $N_{f}=1$.

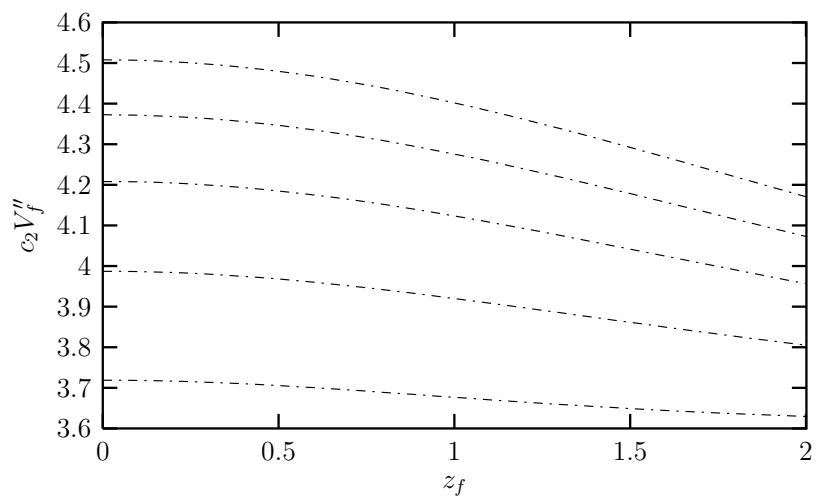

Figure 11: The dependence on $z_{f}$ of $c_{2} V_{f}^{\prime \prime}\left(\theta=0, N_{f}=1, z_{f}, \pi / 2<\delta_{f}<\pi\right)$. Here, $\delta_{f}=\pi$ for the top curve, whereas $\delta_{f}=\pi / 2$ for the bottom curve. For the curves in between $\delta_{f}=$ $6 \pi / 10,7 \pi / 10,8 \pi / 10$, from bottom to top, respectively.

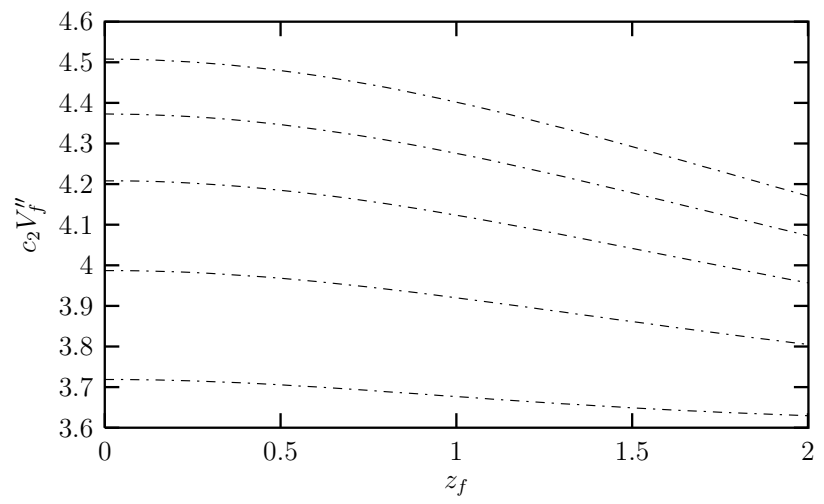

Figure 12: The dependence on $z_{f}$ of $c_{2} V_{f}^{\prime \prime}\left(\theta=\pi, N_{f}=1, z_{f}, 0<\delta_{f}<\pi / 2\right)$. Here, $\delta_{f}=0$ for the top curve, whereas $\delta_{f}=\pi / 2$, for the bottom curve. For the curves in between $\delta_{f}=$ $2 \pi / 10,3 \pi / 10,4 \pi / 10$, from bottom to top, respectively. 
Note that the plots in Figure 11 and Figure 12 are identical despite the fact that the intervals for $\delta_{f}$ are different. This is due to the fact that $V_{f}^{\prime \prime}\left(\theta=0, N_{f}, z_{f}, \delta_{f}\right) \rightarrow V_{f}^{\prime \prime}\left(\theta=\pi, N_{f}, z_{f}, \delta_{f}\right)$ under the transformation $\delta_{f} \rightarrow \delta_{f}-\pi$.

A comparative analysis of Figure 11 and Figure 12 shows that $V_{f}^{\prime \prime}\left(\theta, N_{f}=1, z_{f}, \delta_{f}\right)$ is always positive independent of $z_{f}$; that is there are no critical values for $z_{f}$.

Now, we would like to study the behaviour of $V_{f}(\theta)$ under the variations of $z_{f}$. In Figure 13, we have plotted $V_{f}\left(\theta, z_{f}\right)$ for selected values of $\delta_{f}$ from the region in which $\theta=0$, and $\pi$ are global minima for $z_{f}=0$, respectively.

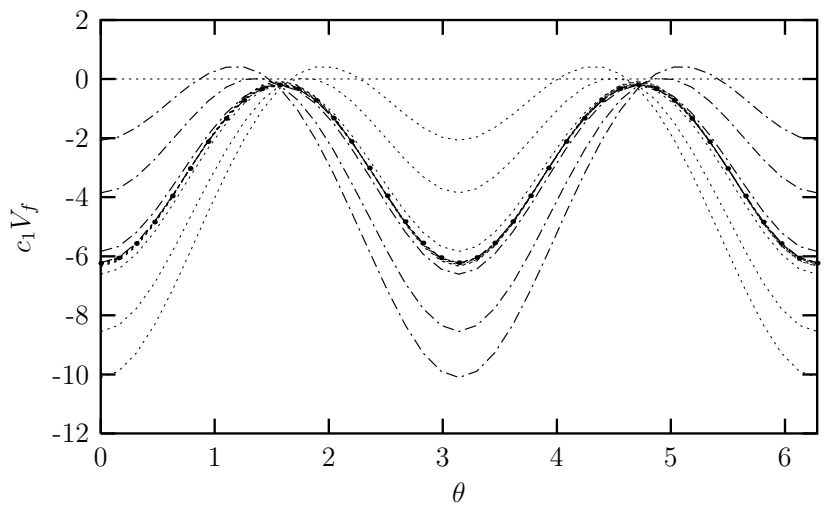

Figure 13: The dependence on $\theta$ of $c_{1} V_{f}\left(\theta, N_{f}=1, z_{f}, \delta_{f}\right)$. Here, $\delta_{f}=0$, and $\delta_{f}=\pi$ are represented by the dot-dashed curves, and dotted curves, respectively. The dot-dashed curves (with $\delta_{f}=0$ ), from bottom to top, are for $z_{f}=0, z_{f}=2$, and $z_{f}=5$, whereas the dotted curves (with $\delta_{f}=\pi$ ), from top to bottom, are for $z_{f}=0, z_{f}=2$, and $z_{f}=5$. When $z_{f}=8$, all the curves coagulate to the same limiting degenerate minima curve (shown by dots in the middle), which always happens at $\delta_{f}=\pi / 2$.

We would like to note that, in Figure 13 we have shown the curves for the values of $z_{f}=0$, $z_{f}=2$, and $z_{f}=5$ only, as they are very densly packed and very difficult to distinguish from each other in the region $5<z_{f}<8$.

We have observed that for $z_{f}=8$, all the curves coagulate to the same limiting curve, corresponding to the degenerate minima $\left(\delta_{f}=\pi / 2\right)$.

Thus, $z_{f}=8$ emerges as some sort of critical value, not in the sense that we move from one global minimum to another, when we cross it; but which ever local minimum we start from, we end up with the degenerate minima case, when we reach this value of $z_{f}$. As the local minima are always either one of the $\theta=0, \pi$, then there is no change in the symmetry pattern.

Finally, we look at the variation of $V_{f}^{\prime \prime}\left(\theta_{m}, N_{f}, z_{f}, \delta_{f}\right)$ in the specific intervals of $\delta_{f}$ found above, for $N_{f}=4$ : For this purpose, in Figure 14 and Figure 15 we analyze the dependence of $c_{2} V_{f}^{\prime \prime}\left(\theta=0, N_{f}=4, z_{f}, \delta_{f}\right)$ on $z_{f}$ in the $\delta_{f}^{c_{2}}=2.61<\delta_{f}<\pi$ and $0<\delta_{f}<\delta_{f}^{c_{1}}=0.53$ intervals, respectively, for selected values of $\delta_{f}$.

Note that Figure 14 and Figure 15 show the same symmetry behaviour we mentioned above, in relation to the Figure 11 and Figure 12.

A comparative analysis of Figure 14 and Figure 15 suggest that the variation of $V_{f}^{\prime \prime}\left(\theta, N_{f}=\right.$ $\left.4, z_{f}, \delta_{f}\right)$ at $\theta=0, \pi$ with respect to $z_{f}$, show similar behaviour to those of $N_{f}=1,2$. Thus, once we restrict $\delta_{f}$ to the allowed range in this case, mass does not play any further role.

In summary the above detailed analysis shows that fundamental fermions do not break $\mathrm{SU}(2)$ symmetry, irrespective of the values of the parameters $\delta_{f}, z_{f}$, and $N_{f}$. 


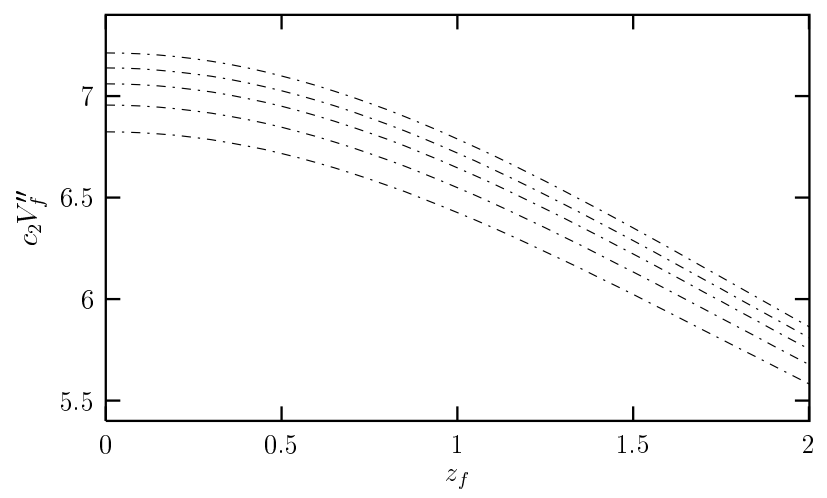

Figure 14: The dependence on $z_{f}$ of $c_{2} V_{f}^{\prime \prime}\left(\theta=0, N_{f}=4, z_{f}, \delta_{f}^{c_{2}}<\delta_{f}<\pi\right)$. Here, $\delta_{f}=\pi$ for the top curve, whereas $\delta_{f}=2.61$, for the bottom curve. For the curves in between, $\delta_{f}=2.91,2.81$, 2.71 , from bottom to top, respectively.

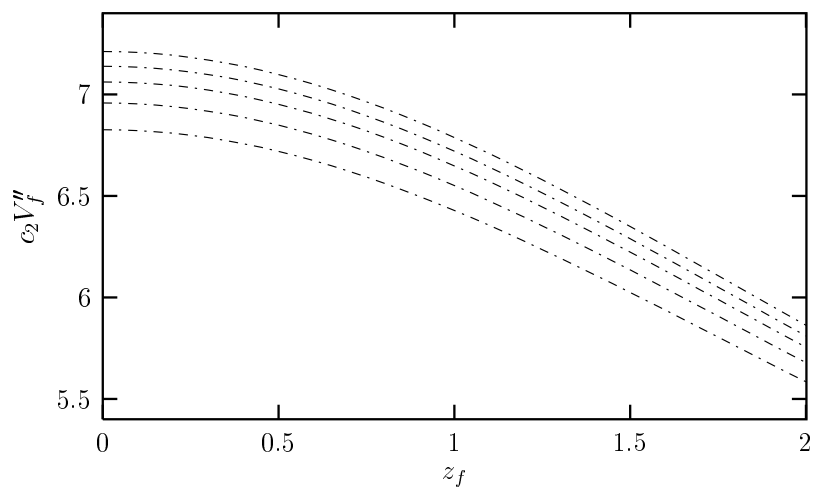

Figure 15: The dependence on $z_{f}$ of $c_{2} V_{f}^{\prime \prime}\left(\theta=\pi, N_{f}=4, z_{f}, 0<\delta_{f}<\delta_{f}^{c_{1}}\right)$. Here, $\delta_{f}=0$ for the top curve, whereas $\delta_{f}=0.53$, for the bottom curve. For the curves in between, $\delta_{f}=0.23,0.33$, 0.43 , from top to bottom, respectively.

\section{$5 \quad$ Fundamental and Adjoint Fermions with equal masses}

With these inputs in mind, let us look at the general case where there are $N_{a}$ massive adjoint fermions, and $N_{f}$ massive fundamental fermions with equal masses $\left(z_{a}=z_{f}=z\right)$.

The potential is given by:

$$
\begin{aligned}
V_{a f}\left(\theta, N_{a}, N_{f}, z, \delta_{a}, \delta_{f}\right) & =\frac{1}{c_{1}} \sum_{n=1} \frac{1}{n^{5}}\left\{\left[-3+4 N_{a} F(z n) \cos n \delta_{a}\right](1+\cos 2 n \theta)\right. \\
& \left.+4 N_{f} F(z n) \cos n \delta_{f} \cos n \theta\right\}
\end{aligned}
$$

The most trivial roots of $V_{a f}^{\prime}$ are $\theta=0, \pi$. In principle, there could be non-trivial roots of $V_{a f}^{\prime}=0$ as well, depending on the values of the parameters; we checked this numerically, and analytically, and have shown that there are no other minima. Again, as in the previous special cases we look at the two special limits, namely $z_{a}=z_{f} \rightarrow \infty$, and $z_{a}=z_{f} \rightarrow 0$. 
One first notes that for $m_{a}=m_{f} \rightarrow \infty, \quad F(z n) \rightarrow 0$ which means $V \rightarrow V_{\text {pure gauge, as }}$ all fermions, adjoint and fundamental, decouple (thus, as before $\theta=0[\bmod \pi]$ is an absolute minimum). For $m_{a}=m_{f} \rightarrow 0, F(z n) \rightarrow 1$, and

$$
\begin{aligned}
c_{2} V_{a f}^{\prime \prime}\left(\theta=0, N_{a}, N_{f}, z=0, \delta_{a}, \delta_{f}\right) & =\sum_{n=1} \frac{1}{n^{3}}\left(3-4 N_{a} \cos n \delta_{a}-N_{f} \cos n \delta_{f}\right), \\
c_{2} V_{a f}^{\prime \prime}\left(\theta=\pi, N_{a}, N_{f}, z=0, \delta_{a}, \delta_{f}\right) & =\sum_{n=1} \frac{1}{n^{3}}\left(3-4 N_{a} \cos n \delta_{a}-N_{f}(-1)^{n} \cos n \delta_{f}\right) .
\end{aligned}
$$

To determine the ranges of $\delta_{f}, \delta_{a}$, we first plot the $\delta_{f}-\delta_{a}$ region, for which $c_{2} V_{a f}^{\prime \prime}\left(\theta=0, N_{a}, N_{f}, z=\right.$ $\left.0, \delta_{a}, \delta_{f}\right)>0$ in Figure 16, when $N_{a}=N_{f}=1$.

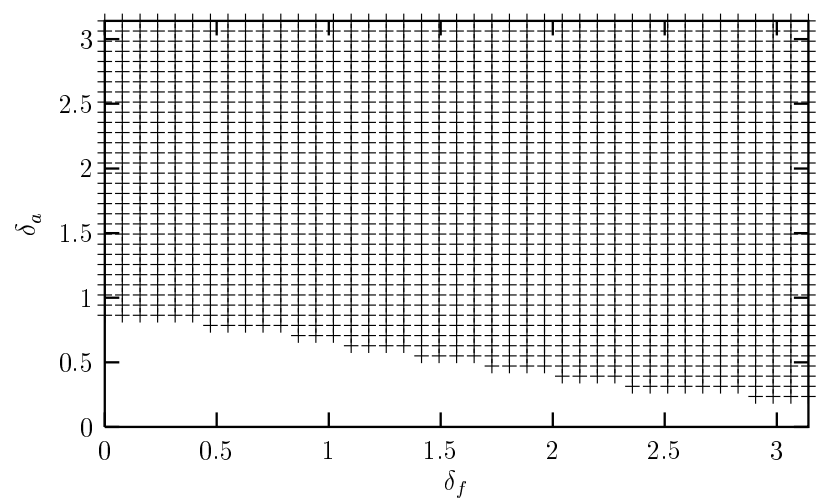

Figure 16: The $\delta_{f}-\delta_{a}$ region, for which $c_{2} V_{a f}^{\prime \prime}\left(\theta=0, N_{a}=N_{f}=1, z=0, \delta_{a}, \delta_{f}\right)>0$.

As can be observed from Figure 16 that the lower bound of $\delta_{a}$ ranges from $\delta_{a}=3 \pi / 40$, up to $\delta_{a}=\pi / 4$, when $\delta_{f}$ changes from 0 to $\pi$. One notes that the lower bound of $\delta_{f}$ ranges from $3 \pi / 20$ to $37 \pi / 40$, in the $3 \pi / 40 \lesssim \delta_{a} \lesssim \pi / 4$, interval. On the other hand, for $10 \pi / 40<\delta_{a}<\pi$, there is no constraint on $\delta_{f}$; That is, all values of $\delta_{f}$ are allowed for $\delta_{a}>10 \pi / 40$.

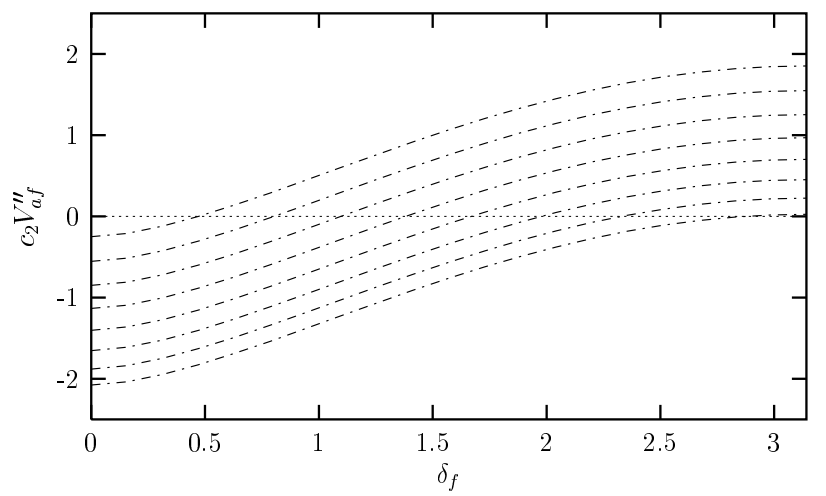

Figure 17: The dependence on $\delta_{f}$ of $c_{2} V_{a f}^{\prime \prime}\left(\theta=0, N_{a}=N_{f}=1, z=0, \delta_{a}, \delta_{f}\right)$, for $3 \pi / 40 \lesssim \delta_{a} \lesssim$ $\pi / 4$. Here, $\delta_{a}=\pi / 4$ for the top curve, whereas $\delta_{a}=3 \pi / 40$, for the bottom curve. In the remaining portion of the parameter space, namely $\delta_{a}<3 \pi / 40$, and $\delta_{a}>\pi / 4, c_{2} V_{a f}^{\prime \prime}$ does not change sign.

In Figure 17 we plot $c_{2} V_{a f}^{\prime \prime}\left(\theta=0, N_{a}=N_{f}=1, z=0, \delta_{a}, \delta_{f}\right)$ with respect to $\delta_{f}$, for the set of values $3 \pi / 40 \lesssim \delta_{a} \lesssim \pi / 4$, for which $c_{2} V_{a f}^{\prime \prime}\left(\theta=0, N_{a}=N_{f}=1, z=0, \delta_{a}, \delta_{f}\right)$ was changing sign in Figure 16. Here, $\delta_{a}=\pi / 4$ for the top curve, whereas $\delta_{a}=3 \pi / 40$ for the bottom curve. 
As can be seen from Figure 17 that when $\delta_{a}=\pi / 4, c_{2} V_{a f}^{\prime \prime}\left(\theta=0, N_{a}=N_{f}=1, z=0, \delta_{a}, \delta_{f}\right)$ changes sign at $\delta_{f}=3 \pi / 20$, whereas for $\delta_{a}=3 \pi / 40$, the lower bound on $\delta_{f}$ moves to $\delta_{f}=37 \pi / 40$. For $\delta_{a}>\pi / 4, c_{2} V_{a f}^{\prime \prime}\left(\theta=0, N_{a}=N_{f}=1, z=0, \delta_{a}, \delta_{f}\right)$ does not change sign for any value of $\delta_{f}$, which is consistent with Figure 16.

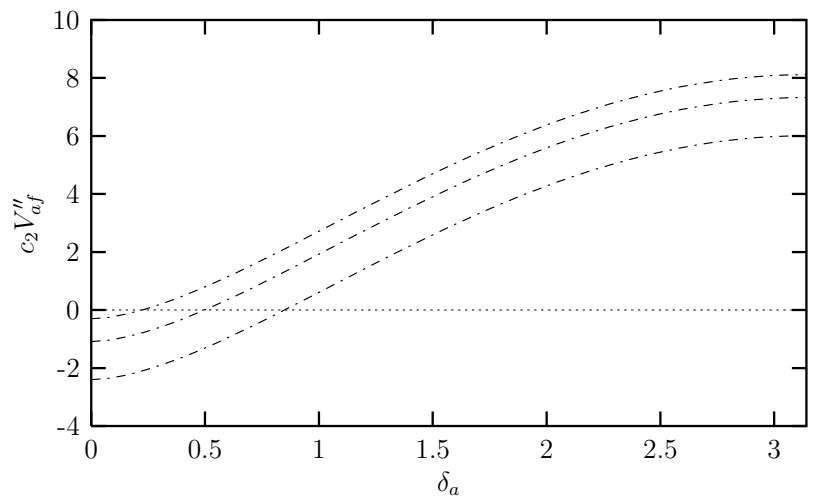

Figure 18: The dependence on $\delta_{a}$ of $c_{2} V_{a f}^{\prime \prime}\left(\theta=0, N_{a}=N_{f}=1, z=0, \delta_{a}, \delta_{f}\right)$. Here, $\delta_{f}=\pi$, $\delta_{f}=\pi / 2$ and $\delta_{f}=0$, for the top, middle, bottom curves, respectively. $c_{2} V_{a f}^{\prime \prime}$ changes sign for all values of $\delta_{f}$; the critical values of $\delta_{a}$ decrease with increasing values of $\delta_{f}$.

In Figure 18, we analyze the dependence on $\delta_{a}$ of $c_{2} V_{a f}^{\prime \prime}\left(\theta=0, N_{a}=N_{f}=1, z=0, \delta_{a}, \delta_{f}\right)$ for given values of $\delta_{f}$, namely $\delta_{f}=\pi$ (top curve), $\delta_{f}=\pi / 2$ (middle curve), and $\delta_{f}=0$ (bottom curve).

As can be seen from Figure 18, $c_{2} V_{a f}^{\prime \prime}(\theta=0)$ changes sign for all values of $\delta_{f}$, whereas the lower bound on $\delta_{a}$ moves from $\pi / 4$ to $3 \pi / 40$, with the increasing values of $\delta_{f}$. For instance, when $\delta_{f}=0$ the lower bound on $\delta_{a}$, at which the $c_{2} V_{a f}^{\prime \prime}$ changes sign, is $\delta_{a}=\pi / 4$, whereas for $\delta_{f}=\pi$, it is $\delta_{a}=3 \pi / 40$, which is consistent with Figure 16 .

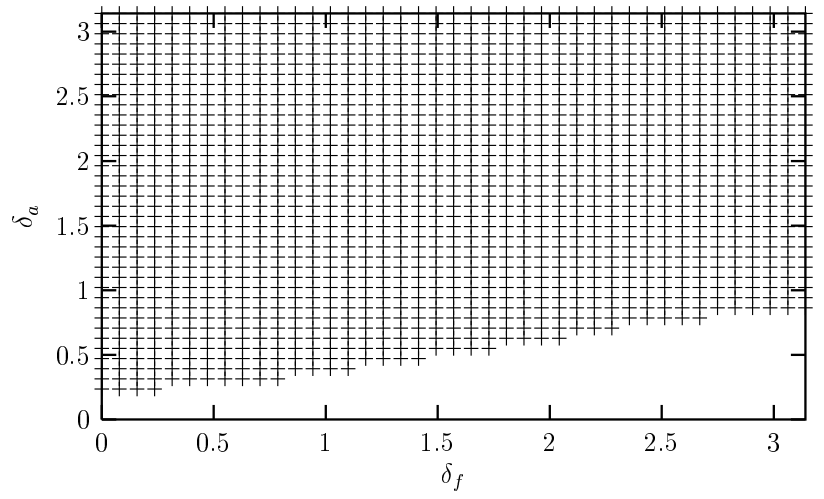

Figure 19: The $\delta_{f}-\delta_{a}$ region, for which $V_{a f}^{\prime \prime}\left(\theta=\pi, N_{a}=N_{f}=1, z=0, \delta_{a}, \delta_{f}\right)>0$.

In Figure 19, we plot the $\delta_{f}-\delta_{a}$ region, for which $c_{2} V_{a f}^{\prime \prime}\left(\theta=\pi, N_{a}=N_{f}=1, z=0, \delta_{a}, \delta_{f}\right)>0$. Here, all values of $\delta_{f}$ from 0 to $\pi$ are allowed above the critical value $\delta_{a}>\pi / 4$, as was the case in Figure 16, also.

In Figure 20, we plot $c_{2} V_{a f}^{\prime \prime}\left(\theta=\pi, N_{a}=N_{f}=1, z=0, \delta_{a}, \delta_{f}\right)$ with respect to $\delta_{f}$, for the set of values $3 \pi / 40 \lesssim \delta_{a} \lesssim \pi / 4$, in the region where $c_{2} V_{a f}^{\prime \prime}\left(\theta=\pi, N_{a}=N_{f}=1, z=0, \delta_{a}, \delta_{f}\right)$ 
was changing sign in Figure 19. In Figure 20, the top and the bottom curves represent $\delta_{a}=\pi / 4$, and $\delta_{a}=3 \pi / 40$, respectively. One notes that $c_{2} V_{a f}^{\prime \prime}\left(\theta=\pi, N_{a}=N_{f}=1, z=0, \delta_{a}, \delta_{f}\right)$ does not change sign in the remaining portion of the $\delta_{a}$-parameter space (namely, for $\delta_{a}<3 \pi / 40$, and $\left.\delta_{a}>\pi / 4\right)$.

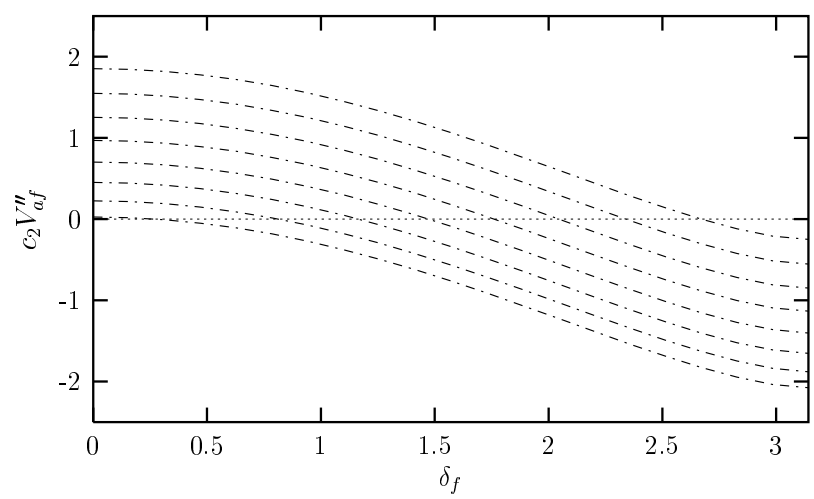

Figure 20: The dependence on $\delta_{f}$ of $c_{2} V_{a f}^{\prime \prime}\left(\theta=\pi, N_{a}=N_{f}=1, z=0, \delta_{a}, \delta_{f}\right)$, for the set of values $3 \pi / 40 \lesssim \delta_{a} \lesssim \pi / 4$. Here, $\delta_{a}=\pi / 4$ for the top curve, whereas $\delta_{a}=3 \pi / 40$, for the bottom curve. In the remaining portion of the $\delta_{a}$-parameter space $c_{2} V_{a f}^{\prime \prime}$ does not change sign.

In Figure 21, we analyze the dependence on $\delta_{a}$ of $c_{2} V_{a f}^{\prime \prime}\left(\theta=\pi, N_{a}=N_{f}=1, z=0, \delta_{a}, \delta_{f}\right)$ for given values of $\delta_{f}$ when $N_{f}=N_{a}=1$. In the Figure, the top, middle, bottom curves represent $\delta_{f}=0, \delta_{f}=\pi / 2$ and $\delta_{f}=\pi$, respectively. Similar to observations made for Figure 20, when $\delta_{f}=\pi$, the potential changes sign at $\delta_{f}=\pi / 4$, whereas for $\delta_{f}=0$, the lower bound on $\delta_{a}$ is $\delta_{f}=3 \pi / 40$, which is consistent with Figure 19 .

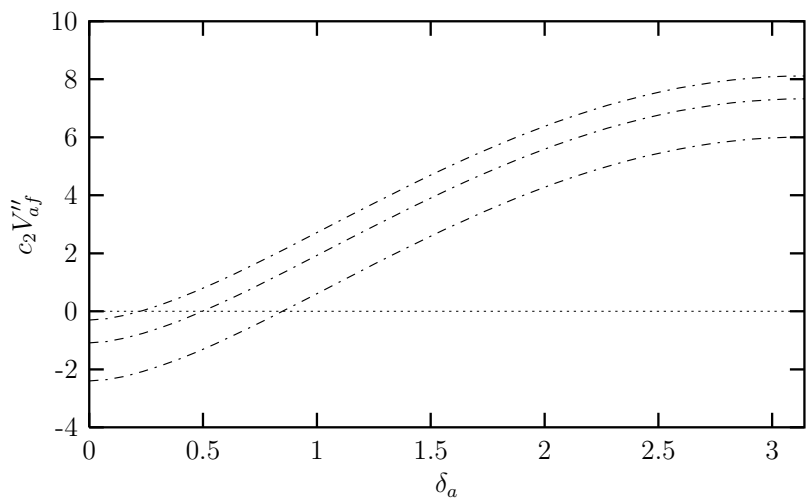

Figure 21: The dependence on $\delta_{a}$ of $c_{2} V_{a f}^{\prime \prime}\left(\theta=0, N_{a}=N_{f}=1, z=0, \delta_{a}, \delta_{f}\right)$. Here, $\delta_{f}=\pi$, $\delta_{f}=\pi / 2$ and $\delta_{f}=0$, for the top, middle and the bottom curves, respectively.

We have to next check the region of $\delta_{a}-\delta_{f}$ for which $\theta=0, \pi$ are the absolute minima. For this purpose, in Figure 22 we have plotted $c_{2} V_{a f}\left(\theta, N_{a}=N_{f}=1, z=0, \delta_{a}, \delta_{f}\right)$, with respect to $\theta$ for selected values of $\delta_{a}>\delta_{a}^{c r}=\pi / 4$, identified in Figure 16 and Figure 19 for both $\theta=0$, and $\pi$.

It is seen from Figure 22 that $\theta=0$ is the absolute minimum for $\pi / 2<\delta_{f}<\pi$, whereas $\theta=\pi$ is the absolute minimum for $0<\delta_{f}<\pi / 2$, as in the pure fundamental fermions case. Note that these are degenerate at $\delta_{f}=\pi / 2$. However, as before irregardless of which one of $\theta=0, \pi$ are the 


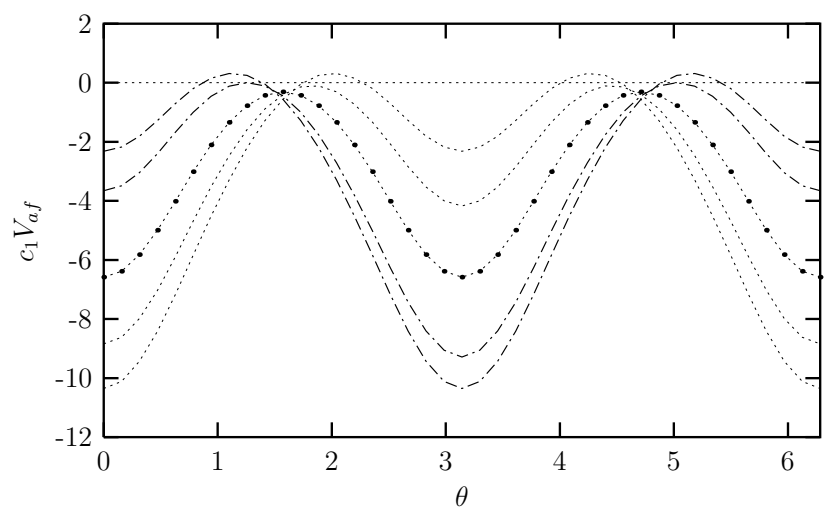

Figure 22: The dependence of $c_{1} V_{a f}\left(\theta, N_{a}=N_{f}=1, z=0, \delta_{a}=\pi / 2, \delta_{f}\right)$ on $\theta$. Here, the dotted-line in the middle is for $\delta_{f}=\pi / 2$. The upper two lines, from top to bottom, are for $\delta_{f}=\pi$, and $\delta_{f}=7 \pi / 10$, respectively, chosen in the $\pi / 2<\delta_{f}<\pi$ interval. The lower two lines are for, from bottom to top, $\delta_{f}=0$, and $\delta_{f}=\pi / 4$, respectively, chosen in the $0<\delta_{f}<\pi / 2$ interval.

absolute minima, the symmetry is unbroken, provided that $\delta_{a}>\delta_{a}^{c r}=\pi / 4$.

Similar analysis can be made for $c_{1} V_{a f}\left(\theta, N_{a}=N_{f}=1, z=0, \delta_{a}, \delta_{f}=\pi / 2\right)$, for selected values of $\delta_{a}$, chosen from the region $\delta_{a}>\delta_{a}^{c r}=\pi / 4$. We see that in this case $\theta=0, \pi$ are the degenerate absolute minima, which is consistent with the remarks of Figure 16 and Figure 19.

Next, we would like to adress the issue of stability of these absolute minima, we have found for the massless case, under the variations of $\mathrm{z}$.

We have previously observed that there were critical values of $\mathrm{z}$, at which symmetry pattern changed, for the adjoint case. But in the fundamental fermions case the mass did not play any role on the symmetry pattern. In the present case, that is when the fundamental and adjoint fermions exist together (with equal masses), we would like to check which behaviour of the previous special cases would be carried over.

We get from Eq. (22):

$$
\begin{aligned}
c_{2} V_{a f}^{\prime \prime}\left(\theta=0, N_{a}, N_{f}, z, \delta_{a}, \delta_{f}\right) & =\sum_{n=1} \frac{1}{n^{3}}\left(3-4 N_{a} F(z n) \cos n \delta_{a}\right. \\
& \left.-N_{f} F(z n) \cos n \delta_{f}\right), \\
c_{2} V_{a f}^{\prime \prime}\left(\theta=\pi, N_{a}, N_{f}, z, \delta_{a}, \delta_{f}\right) & =\sum_{n=1} \frac{1}{n^{3}}\left(3-4 N_{a} F(z n) \cos n \delta_{a}\right. \\
& \left.-N_{f}(-1)^{n} F(z n) \cos n \delta_{f}\right) .
\end{aligned}
$$

In Figure 23, and Figure 24, we plot the 3-dimensional graphs depicting $c_{2} V_{a f}^{\prime \prime}\left(\theta=0, N_{a}=\right.$ $\left.N_{f}=1, z, \delta_{a}, \delta_{f}\right)$, against $\delta_{a}-z$, and $\delta_{f}-z$, when $\delta_{a}-\delta_{f}$ are restricted to the region where $\theta=0$ was a minimum in the massless case.

In Figure 23, we obtain 3-dimensional surfaces for each value of $\delta_{f}$, which we choose within the interval $[0, \pi]$. For instance the top surface corresponds to $\delta_{f}=\pi$, whereas the bottom one represents $\delta_{f}=0$. One notes that for $\delta_{f}=0$, the lowest allowed bound of $\delta_{a}$ is $\delta_{a}=11 \pi / 40$, consistent with Figure 16. 


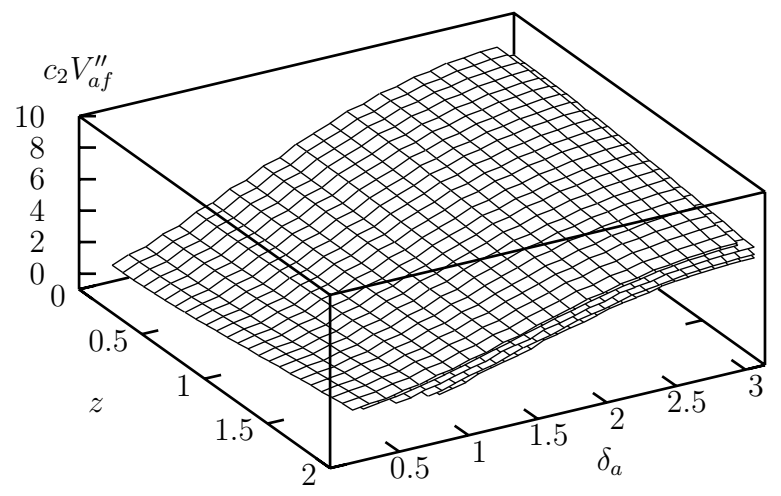

Figure 23: The dependence of $c_{2} V_{a f}^{\prime \prime}\left(\theta=0, N_{a}=N_{f}=1, z, \delta_{a}, \delta_{f}\right)$ on $\delta_{a}$ and z, for selected values of $\delta_{f}$. Here, $\delta_{a}$ and $\delta_{f}$ values are restricted to the shaded region in Figure 16.

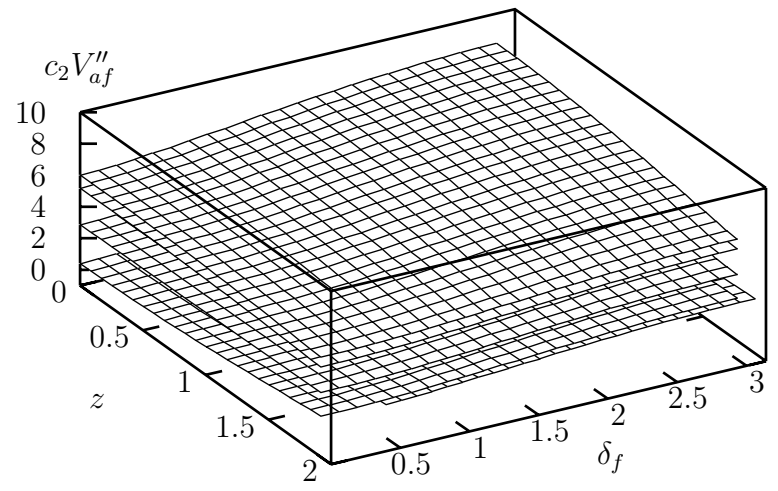

Figure 24: The dependence of $c_{2} V_{a f}^{\prime \prime}\left(\theta=0, N_{a}=N_{f}=1, z, \delta_{a}, \delta_{f}\right)$ on $\delta_{f}$ and z, for selected values of $\delta_{a}$. Here, $\delta_{a}$ and $\delta_{f}$ values are restricted to the shaded region in Figure 16.

Similarly, each three dimensional surface in Figure 24 corresponds to a discrete value of $\delta_{a}$ changing in the $[0, \pi]$ interval. Here, the bottom surface represents $\delta_{a}=\pi / 4$, at which case the lowest allowed bound on $\delta_{f}$ is $\delta_{f}=3 \pi / 20$, as in Figure 16 .

A comparative analysis of Figure 23 and Figure 24 shows that $c_{2} V_{a f}^{\prime \prime}\left(\theta=0, N_{a}=N_{f}=\right.$ $\left.1, z, \delta_{a}, \delta_{f}\right)$, does not change sign with the variations of $\mathrm{z}$, when $\delta_{a}$, and $\delta_{f}$ are restricted to the shaded region in Figure 16. That is $\theta=0$ remains as a minimum independent of the values of $\mathrm{z}$.

To address the stability issue of the minimum $\theta=\pi$, we plotted a similar set of 3 -dimensional graphs depicting $c_{2} V_{a f}^{\prime \prime}\left(\theta=\pi, \quad N_{a}=N_{f}=1, z, \delta_{a}, \delta_{f}\right)$, against $\delta_{a}-z$ in Figure 25 , and $\delta_{f}-z$ in Figure 26, where $\delta_{a}$ and $\delta_{f}$ values are restricted to the shaded region in Figure 19. 


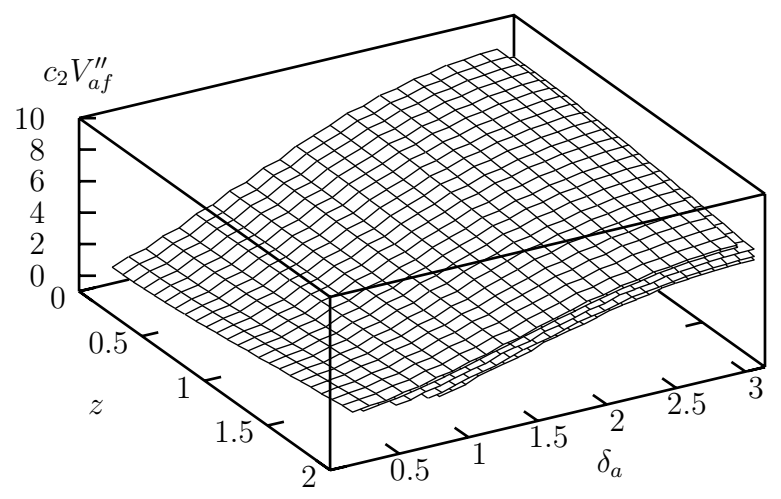

Figure 25: The dependence of $c_{2} V_{a f}^{\prime \prime}\left(\theta=\pi, N_{a}=N_{f}=1, z, \delta_{a}, \delta_{f}\right)$, on $\delta_{a}$ and z, for selected values of $\delta_{f}$. Here, $\delta_{a}$ and $\delta_{f}$ values are restricted to the shaded region in Figure 19.

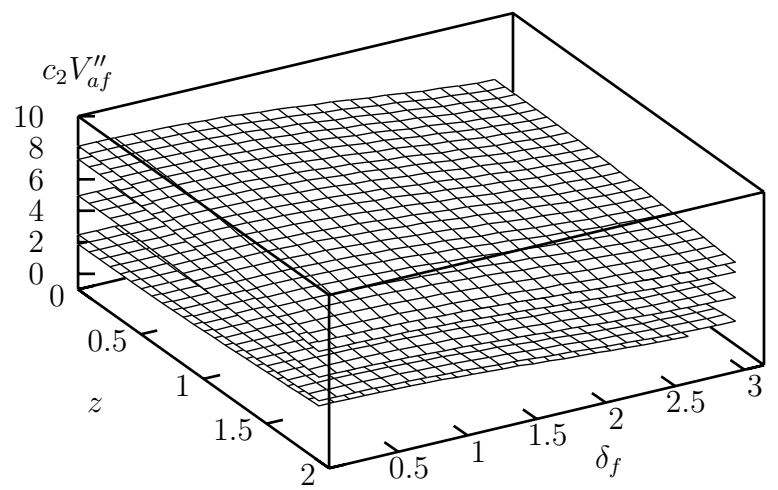

Figure 26: The dependence of $c_{2} V_{a f}^{\prime \prime}\left(\theta=\pi, N_{a}=N_{f}=1, z, \delta_{a}, \delta_{f}\right)$, on $\delta_{f}$ and z, for selected values of $\delta_{a}$. Here, $\delta_{a}$ and $\delta_{f}$ values are restricted to the shaded region in Figure 19.

In Figure 25, the top surface corresponds to $\delta_{f}=0$, and the bottom one represents $\delta_{f}=\pi$, whereas in Figure 26, the top surface corresponds to $\delta_{a}=\pi$, and the bottom one to $\delta_{a}=\pi / 4$.

A comparative analysis of Figure 25 and Figure 26 shows that $c_{2} V_{a f}^{\prime \prime}\left(\theta=\pi, N_{a}=N_{f}=\right.$ $\left.1, z, \delta_{a}, \delta_{f}\right)$ does not change sign, and always stays positive, independent of the values of $\mathrm{z}$, in the allowed region of $\delta_{a}-\delta_{f}$.

Now, we would like to investigate the special case in which $\theta=0$ and $\theta=\pi$ minima are degenerate which can be checked easily to occur for $\delta_{f}=\pi / 2$, and $\delta_{a}>\delta_{a}^{c r}$. The result is depicted in Figure 27, where we plot $c_{2} V_{a f}^{\prime \prime}\left(\theta=0[\bmod \pi], N_{a}=N_{f}=1, z, \delta_{a}, \delta_{f}\right)$ with respect to z for $\delta_{f}=\pi / 2$, and for the selected set of values of $\delta_{a}$ within the allowed range; $\delta_{a}>\delta_{a}^{c r}=\pi / 4$. Namely, we choose $\delta_{a}=\pi$ (top curve), $\delta_{a}=3 \pi / 2$ (middle curve), $\delta_{a}=\pi / 2$ (bottom curve).

We see that for this special regime of the parameters, namely $\delta_{f}=\pi / 2, \delta_{a}>\delta_{a}^{c r}, V_{a f}^{\prime \prime}(\theta=0, \pi)$ is always positive (does not change sign) with the variations of $\mathrm{z}$. 


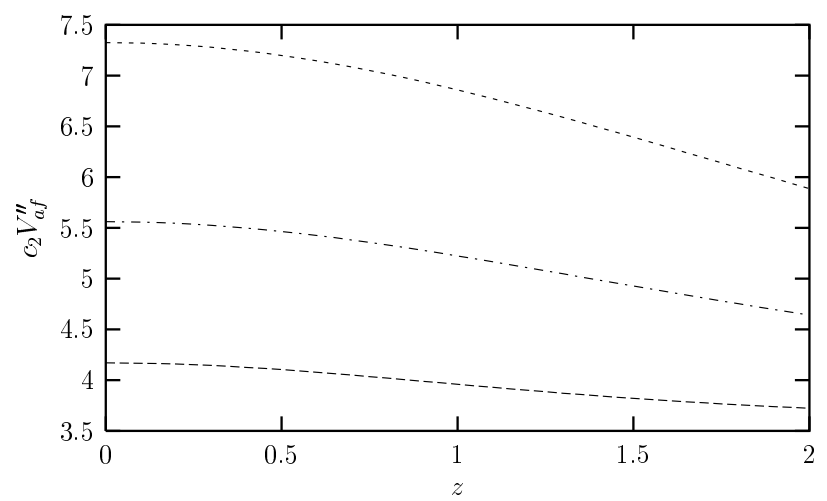

Figure 27: The dependence on $\mathrm{z}$ of $c_{2} V_{a f}^{\prime \prime}\left(\theta=0[\bmod \pi], N_{a}=N_{f}=1, z, \delta_{a}, \delta_{f}\right)$ when $\delta_{f}=\pi / 2$, for the selected set of values of $\delta_{a}: \delta_{a}=\pi$ (top curve), $\delta_{a}=3 \pi / 2$ (middle curve), $\delta_{a}=\pi / 2$ (bottom curve).

Lastly, we would like to study the behaviour of the effective potential under the variations of z. For this purpose, in Figure 28, we have plotted $V_{a f}(\theta, \mathrm{z})$ for selected characteristic values of $\delta_{a}$ and $\delta_{f}$ picked from Figure 22 (which in turn identified from Figure 16 and 19), defining the allowed regions of the parameter space for the local minima $\theta_{m}=0, \pi$.

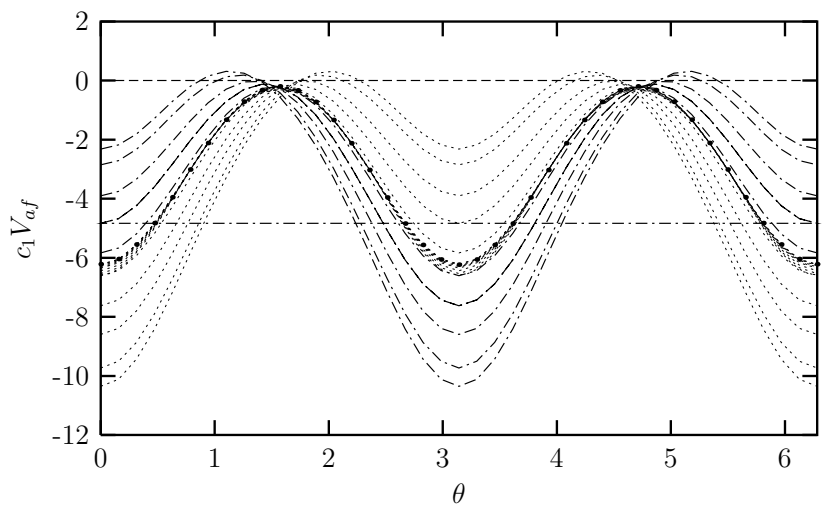

Figure 28: The dependence on $\theta$ of $V_{a f}$ when $N_{f}=1, N_{a}=1$, for the cases $\delta_{a}=\pi / 2, \delta_{f}=\pi / 2$ (shaded region), $\delta_{a}=\pi / 2, \delta_{f}=0$ (dot-dashed curves), $\delta_{a}=\pi / 2, \delta_{f}=\pi$ (dotted curves). Here, the dot-dashed lines, from bottom to top, are for $z=0, z=1, z=2, z=3, z=5$. The dotted lines, from top to bottom, are for $z=0, z=1, z=2, z=3, z=5$. Again when $z_{f}=8$, all the curves coagulate to the same limiting degenerate minima curve (shown by dots on the edge of the shaded region), which always happens at $\delta_{f}=\pi / 2$, irregardless of the values of $\delta_{a}$.

We see from Figure 28 that, for the pair $\delta_{a}=\pi / 2, \delta_{f}=\pi$, we get the dotted curves for the values of $z=0, z=1, z=2, z=3, z=5$, from top to bottom, with the global minimum $\theta_{m}=0$. Similarly, for $\delta_{a}=\pi / 2, \delta_{f}=0$, we obtain the dot-dashed curves for the values of $z=0, z=1$, $z=2, z=3, z=5$, from bottom to top, with the global minimum $\theta_{m}=\pi$.

We see that these two groups of curves (with $\theta_{m}=0$, and $\theta_{m}=\pi$, respectively) all coagulate to the limiting curve shown by dots on the edge of the shaded region, which corresponds to the degenerate minima case (for $\delta_{f}=\pi / 2$ irregardless of the values of $\delta_{a}$ ). 
Thus $z=8$ emerges as some critical value, not in the sense that we move from one global minimum to another, when we cross this value; but whichever local minimum we start from (in the massless case) we end up with the degenerate minima case when we reach this value of $z$, as in the pure fundamental fermions case. As the absolute minima are always either one of the $\theta=0, \pi$, then there is no change in symmetry pattern as a consequence of this rather intricate dynamical phenomenon.

The above detailed analysis shows that the absolute minima of the massless case, namely $\theta=0$ or $\theta=\pi$ are unaffected, and the $\mathrm{SU}(2)$ symmetry is unbroken by the fermion masses, a behaviour we are familiar with from the special case of pure fundamental fermions. Furthermore, this result is self-consistent, as we have the same minima and thus the same symmetry pattern in the $m \rightarrow 0$, and $m \rightarrow \infty$ cases. That is the fundamental fermions play a more dominant role than the adjoint ones in determining the symmetry pattern.

\section{Conclusions}

In this work, we have constructed the effective potential for the Wilson loop in the $\mathrm{SU}(2)$ gauge theory with $N_{f}$ massive fundamental and $N_{a}$ massive adjoint fermions on $S^{1} \times M^{4}$ in the one-loop level, assuming periodic boundary condition for the gauge field, and the general boundary conditions for fermions with arbitrary phase, and investigated the symmetry structure of the vacuum.

Our results can be summarized as follows:

(i)For the adjoint fermions, the symmetries of the system depend critically on both the bulk mass and the bc parameters. We have considered the special limits of the general case, namely the regime of Hosotani with massless fermions and arbitrary boundary conditions, that of Takenaga with massive fermions and periodic boundary conditions $\left(\delta_{a}=0\right)$, and that of Davies and McLachan (the simplest of them all) with massless fermions, and periodic boundary conditions. Our predictions are identical to theirs in the corresponding limits. We have further observed an interesting phenomenon, that for a special value of $\delta_{a}$ (namely, $\delta_{a}=0.71$, when $N_{a}=1$ ) both broken and unbroken phases coexist for $z_{a} \leq 0.7$. Further analysis of this phenomenon is postponed to a future work.

(ii) Fundamental fermions can never lead to a spontaneous breakdown of the gauge symmetry irrespective of the values of the parameters $z_{f}, \delta_{f}$, and $N_{f}$.

(iii) When there are fundamental and adjoint fermions together (with equal masses), we first note that in the massless case there are critical values for the boundary condition parameters $\delta_{a}$ and $\delta_{f}$, in deciding the absolute minima. However as these are either one of $\theta_{m}=0, \pi$, the symmetry is intact irregardless of their preferences. Thus there are no critical values for the bc parameters as far as symmetry breaking pattern is concerned. We further checked the role played by the masses on the symmetry pattern. We have observed that the minimum values of the effective potential change with the variations of $\mathrm{z}$, however not as much to change the global minimum from $\theta_{m}=0$ to $\theta_{m}=\pi$, or vice-versa. There is a special value of $\mathrm{z}, z=8$, at which all the curves coagulate to the same limiting degenerate minima (which always happens at $\delta_{f}=\pi / 2$ ). However, as the absolute minima are always either one of the $\theta_{m}=0, \pi$, the $\mathrm{SU}(2)$ symmetry remains intact independent of the masses, provided that the boundary condition parameters are chosen within the allowed region of the massless regime. It is interesting that the fundamental fermions play a more dominant role on the gauge symmetry pattern than the adjoint ones, when they act together, as the result is identical to the pure fundamental fermions case.

As explained in the introduction, one immediate application of the aforementioned compactification is to use $\theta(x)$ as inflaton. The cosmological data require the inflaton potential to be rather smooth and inflaton itself to take super-Planckian values. This necessitates the extension 
of field-theoretic description of Nature into string territory which is hardly acceptable. However, as already pointed out in [8,9] and extended to massive bulk fields in [10], the non-integrable phase $\theta(x)$ is a perfect inflaton candidate due to its shift symmetry ( as implied by the higher dimensional gauge invariance). The novelty provided by our analysis is that possible symmetry breaking parameter domains are identified, and thus, the theory below $1 / R$ might look like either as an Abelian or non-Abelian theory. In each case, experimentally favoured four-dimensional gauge coupling $g_{4}(1 / R) \sim 10^{-3}[8,9]$ experiences different constraints from experimental data at the weak scale.

Another point which might be of phenomenological importance concerns the creation of $\mathrm{Q}$ balls. Indeed, the four-dimensional effective theory for the non-integrable phase possesses either and Abelian or non-Abelian invariance, and in either case its self-interactions generate lumps of $\theta(x)$ matter in which all symmetries are broken $[11,12]$. These lumps of matter are perfect dark matter candidates. Here one notices that such Q-balls differ from the Kaluza-Klein Q-balls of [13] in that the latter rests on the inclusion of all Kaluza-Klein modes whereas the former is based on only $\theta(x)$ which is the zero-mode of $A_{5}(x, y)$. The stability as well as further characteristics of Q-balls of non-integrable phase factor need further analysis of (5).

We would like to thank Durmuş A. Demir for extremely helpful discussions.

\section{References}

[1] Y. Aharonov and D. Bohm, Phys. Rev. 115 (1959) 485.

[2] Y. Hosotani, Phys. Lett. B 126 (1983) 309.

[3] A. Higuchi and L. Parker, Phys. Rev. D 37 (1988) 2853.

[4] A. T. Davies and A. McLachlan, Phys. Lett. B 200 (1988) 305.

[5] Y. Hosotani, Annals Phys. 190 (1989) 233.

[6] K. Takenaga, Phys. Lett. B 570 (2003) 244 [arXiv:hep-th/0305251].

[7] P. Candelas, G. T. Horowitz, A. Strominger and E. Witten, Nucl. Phys. B 258 (1985) 46; J. D. Breit, B. A. Ovrut and G. Segre, Phys. Lett. B 162 (1985) 303.

[8] N. Arkani-Hamed, H. C. Cheng, P. Creminelli and L. Randall, Phys. Rev. Lett. 90 (2003) 221302 [arXiv:hep-th/0301218].

[9] N. Arkani-Hamed, H. C. Cheng, P. Creminelli and L. Randall, JCAP 0307 (2003) 003 [arXiv:hep-th/0302034].

[10] L. Pilo, D. A. J. Rayner and A. Riotto, Phys. Rev. D 68 (2003) 043503 [arXiv:hep-ph/0302087].

[11] S. R. Coleman, Nucl. Phys. B 262 (1985) 263 [Erratum-ibid. B 269 (1986) 744];

[12] A. Kusenko, Phys. Lett. B 404 (1997) 285 [arXiv:hep-th/9704073].

[13] D. A. Demir, Phys. Lett. B 495 (2000) 357 [arXiv:hep-ph/0006344]. 